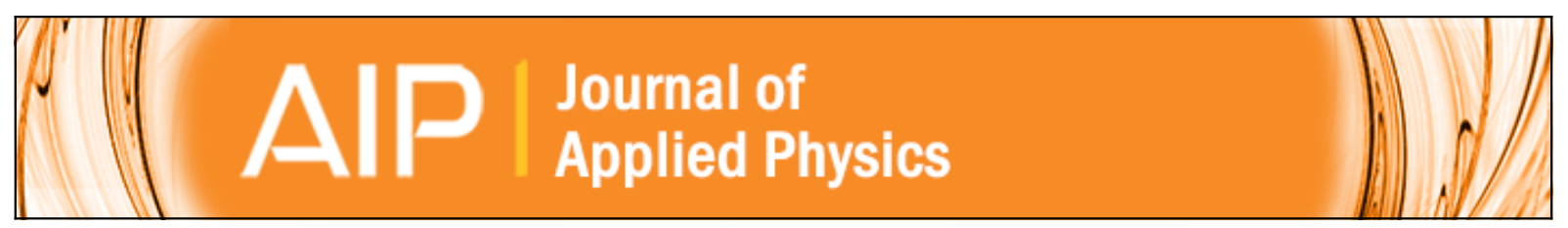

\title{
Periodicity in bimodal atomic force microscopy
}

Chia-Yun Lai, Victor Barcons, Sergio Santos, and Matteo Chiesa

Citation: Journal of Applied Physics 118, 044905 (2015); doi: 10.1063/1.4927733

View online: http://dx.doi.org/10.1063/1.4927733

View Table of Contents: http://scitation.aip.org/content/aip/journal/jap/118/4?ver=pdfcov

Published by the AIP Publishing

\section{Articles you may be interested in}

Selection of higher eigenmode amplitude based on dissipated power and virial contrast in bimodal atomic force microscopy

J. Appl. Phys. 116, 104901 (2014); 10.1063/1.4894837

Non-contact bimodal magnetic force microscopy

Appl. Phys. Lett. 104, 112412 (2014); 10.1063/1.4869353

Enhanced sensitivity and contrast with bimodal atomic force microscopy with small and ultra-small amplitudes in ambient conditions

Appl. Phys. Lett. 103, 231603 (2013); 10.1063/1.4840075

Analysis of the contrast mechanism in bimodal atomic force microscopy combining amplitude modulation and band excitation

J. Appl. Phys. 111, 054909 (2012); 10.1063/1.3692393

Nanotomography with enhanced resolution using bimodal atomic force microscopy

Appl. Phys. Lett. 92, 143107 (2008); 10.1063/1.2907500

\section{HDDEN}

\section{Instruments for Advanced Science}

Contact Hiden Analytical for further details: w www.HidenAnalytical.com E info@hiden.co.uk CLICK TO VIEW our product catalogue

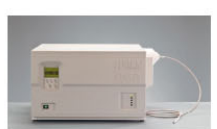

Gas Analysis

dynamic measurement of reaction gas streams catalysis and thermal analysis

molecular beam studies

, dosolled specis probes

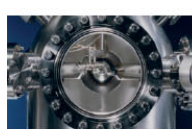

Surface Science

UHVTPD

SIMS end point detection in ion beam etch

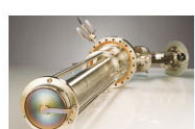

Plasma Diagnostics etch and deposition process reaction etic studies , analysis of neutral and radical species

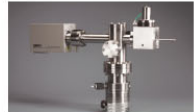

Vacuum Analysis partial pressure measurement and control of process gases reactive sputter process contro , vacuum diagnostics Tacum coating process monitoring 


\title{
Periodicity in bimodal atomic force microscopy
}

\author{
Chia-Yun Lai, ${ }^{1, a)}$ Victor Barcons, ${ }^{2, a)}$ Sergio Santos, ${ }^{1, b)}$ and Matteo Chiesa ${ }^{1}$ \\ ${ }^{1}$ Laboratory for Energy and NanoScience (LENS), Institute Center for Future Energy (iFES), Masdar Institute \\ of Science and Technology, Abu Dhabi, United Arab Emirates \\ ${ }^{2}$ Departament de Disseny i Programació de Sistemes Electrònics, UPC - Universitat Politècnica de Catalunya, \\ Av. Bases, 61, 08242 Manresa (Barcelona), Spain
}

(Received 8 April 2015; accepted 21 July 2015; published online 30 July 2015)

\begin{abstract}
Periodicity is fundamental for quantification and the application of conservation principles of many important systems. Here, we discuss periodicity in the context of bimodal atomic force microscopy (AFM). The relationship between the excited frequencies is shown to affect and control both experimental observables and the main expressions quantified via these observables, i.e., virial and energy transfer expressions, which form the basis of the bimodal AFM theory. The presence of a fundamental frequency further simplifies the theory and leads to close form solutions. Predictions are verified via numerical integration of the equation of motion and experimentally on a mica surface. (C) 2015 AIP Publishing LLC. [http://dx.doi.org/10.1063/1.4927733]
\end{abstract}

\section{INTRODUCTION}

Over the past decades, the field of atomic force microscopy (AFM) has evolved from quasi-static ${ }^{1}$ to dynamic methods $^{2-4}$ and more recently to dynamic methods where multiple frequencies are externally excited. ${ }^{5-8}$ In standard monomodal AFM, the cantilever is externally excited at a single frequency. ${ }^{9}$ When the tip interacts with the nonlinear tip-sample forces, exact multiples of the drive frequency, i.e., its higher harmonics, are excited. ${ }^{9,10}$ Other than in heavily damped environments, however, ${ }^{11}$ and provided the tipsample forces are gentle, ${ }^{12}$ higher harmonics are typically assumed to lie too close to the noise level and are neglected. Then, the amplitude, phase, and mean deflection of the cantilever at the frequency of the drive are the experimental observables. In the multifrequency approach, two ${ }^{13}$ or more ${ }^{7,14,15}$ external drives are employed to excite the cantilever at or near the natural frequencies or eigenmodes of the free cantilever. The simultaneous excitation of two frequencies (see a illustration of bimodal excitation in Fig. 1) was first proposed in 2004 (Ref. 5) and the method is typically known as bimodal AFM. ${ }^{5,15,16}$ Bimodal AFM gives rise to the simultaneous detection of multiple experimental observables and secondary contrast channels, ${ }^{17}$ i.e., the amplitude and phase of the frequency near the higher modal frequencies. These channels enable accessing compositional contrast without the requirement of dissipating energy to the sample, ${ }^{5}$ lead to enhanced resolution, ${ }^{16}$ and provide the means to robustly quantify sample properties. ${ }^{18}$ Still, while attractive for the development of the field, the simultaneous excitation of multiple frequencies at or near the eigenmodes comes at the cost of additional instrumentation, ${ }^{19}$ added complexity to cantilever dynamics ${ }^{20,21}$ and the requirement of interpreting secondary contrast channels. ${ }^{7,14,22,23}$ Furthermore, in order to exploit the potential of bimodal AFM, it is of great interest

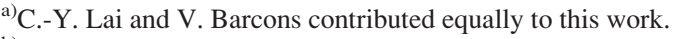

b) Author to whom correspondence should be addressed. Electronic mail: santos_en@yahoo.com.
}

to understand whether the motion is, or can be forced to be, periodic. Periodic motion in this context implies that there is a fundamental frequency the integer multiples of which constitute the full set of higher frequencies present in the signal. In particular, and from a physical point of view, periodicity would imply a repetition in peak ${ }^{24,25}$ and average forces, ${ }^{26}$ and simplicity in terms of quantification of dissipative, i.e., energy dissipation, ${ }^{27}$ and conservative, i.e., viral or frequency shift, ${ }^{20,28}$ interactions. The lack of periodicity on the other hand would imply that these phenomena and expressions repeat only on average. This work deals with periodicity in bimodal AFM and provides a theoretical and experimental discussion on whether periodicity occurs and/ or whether it can be controlled via operational parameters.

\section{RESULTS}

A starting point to discuss periodicity is to consider the higher mode resonant frequencies (modes) of commercial cantilevers. Commercial cantilevers are generally anharmonic in the sense that they are not integer multiples of the fundamental mode. ${ }^{19,29}$ This conclusion can be derived
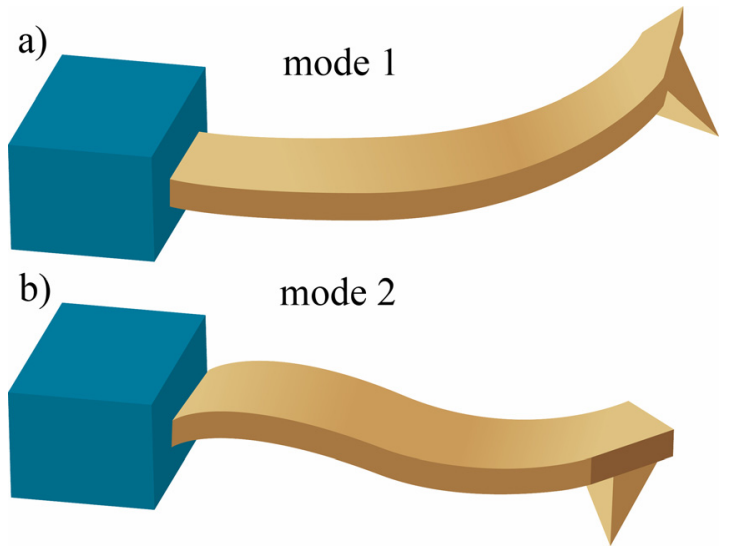

FIG. 1. Illustration of an atomic force micro-cantilever oscillating at the two first eigenmodes. 
analytically ${ }^{30}$ from standard beam theory ${ }^{31}$ and experimentally verified. ${ }^{32}$ Expressions derived from beam theory are typically reported in works dealing with multifrequency AFM. ${ }^{30,33}$ Some groups have also proposed manufacturing harmonic cantilevers ${ }^{29}$ and discussed advantages of harmonicity. ${ }^{34}$ The presence of the probe on the cantilever might further lead to slight deviations from the theory ${ }^{33}$ in real applications. ${ }^{19,35}$ In general, if a signal is periodic, there exists a fundamental frequency $\omega_{\mathrm{F}}$ and integer multiples of this frequency constitute the full set of harmonics present in the signal. According to Fourier analysis, the motion in the vertical axis $\mathrm{z}$ can be written as ${ }^{36}$

$$
z=z_{0}+\sum_{n>0} z_{n}=z_{0}+\sum_{n>0} A_{n} \sin \left(n \omega_{F} t+\phi_{n}\right) .
$$

In the context of AFM, z stands for tip position in the direction normal to the surface of the unperturbed cantilever, $\mathrm{z}_{0}$ is the mean deflection, $n$ is the set of natural numbers, $z_{n}$ is the $\mathrm{n}_{\mathrm{th}}$ harmonic component of motion, and $\mathrm{A}_{\mathrm{n}}$ and $\varphi_{\mathrm{n}}$ are the corresponding $\mathrm{n}_{\mathrm{th}}$ amplitude and phase. It will be shown that the existence and identification of $\omega_{\mathrm{F}}$ make calculations and an interpretation of the data in bimodal AFM simpler. The fundamental period $\mathrm{T}$ can be associated with $\omega_{\mathrm{F}}$ or $2 \pi \mathrm{f}_{\mathrm{F}}$ in (1) as $T=2 \pi / \omega_{\mathrm{F}}$, where $\mathrm{f}_{\mathrm{F}}$ is the fundamental frequency in Hz. $\omega_{\mathrm{F}}$ and $\mathrm{f}_{\mathrm{F}}$ are used interchangeably to refer to fundamental frequency from now on. In bimodal AFM, two external drives are employed to excite the cantilever at frequencies near the modal resonant frequencies $\omega_{(\mathrm{m})}$, where $\mathrm{m}$ stands for mode number, i.e., $\omega_{(1)}$ and $\omega_{(2)}$ in bimodal AFM. Mode numbers are bracketed in this work to distinguish them from harmonic number $\mathrm{n}$ as done elsewhere. ${ }^{23,37}$ The external drive frequencies are $\omega_{\mathrm{D} 1}$ and $\omega_{\mathrm{D} 2} ; \mathrm{D} 1$ and $\mathrm{D} 2$ stand for drives 1 and 2 , respectively. Typically, $\omega_{\mathrm{D} 1} \approx \omega_{(1)}$ and $\omega_{\mathrm{D} 2} \approx \omega_{(2)}$. Nevertheless, there will be times here where $\omega_{\mathrm{D} 1} \neq \omega_{(1)}$ and/or $\omega_{\mathrm{D} 2} \neq \omega_{(2)}$. Furthermore, integer multiples of $\omega_{\mathrm{F}}$ are simply termed as higher harmonics from now on. The key point to note is that higher harmonics imply integer multiples of $\omega_{\mathrm{F}}$ but not necessarily of the external drive frequencies $\omega_{\mathrm{D} 1}$ and/or $\omega_{\mathrm{D} 2}$. Furthermore, from (1), it follows that for the motion to be periodic, $\omega_{\mathrm{D} 1}$ and $\omega_{\mathrm{D} 2}$ are required to be integer multiples of $\omega_{\mathrm{F}}$. Therefore, we write

$$
\omega_{F}=\frac{\omega_{D 1}}{p}=\frac{\omega_{D 2}}{q} \quad \text { where } \mathrm{r}=\mathrm{q} / \mathrm{p} .
$$

In practical terms $\omega_{\mathrm{D} 1}=\mathrm{p} \omega_{\mathrm{F}}$ and $\omega_{\mathrm{D} 1}=\mathrm{q} \omega_{\mathrm{F}}$, where $\mathrm{p}$ and $\mathrm{q}$ are natural numbers that, according to Fourier analysis, ${ }^{36}$ have no common multiples, i.e., $r=q / p$ cannot be further reduced. A main implication of the above discussion, expressions in (1) and (2) and their relationship to bimodal AFM, can now be explicitly stated. Namely, the user's choice of $\omega_{\mathrm{D} 1}$ and $\omega_{\mathrm{D} 2}$ (or choice of cantilever with a given relationship between $\omega_{(1)}$ and $\omega_{(2)}$ if $\omega_{\mathrm{D} 1}=\omega_{(1)}$ and $\left.\omega_{\mathrm{D} 2}=\omega_{(2)}\right)$ dictates the numerical value of the fundamental frequency $\omega_{\mathrm{F}}$ and hence of the fundamental period $\mathrm{T}$, repeats of peak forces per period $2 \pi / \omega_{\mathrm{D} 1}$, and so on. Furthermore, if the relationship between $\omega_{\mathrm{D} 1}$ and $\omega_{\mathrm{D} 2}$ implies incommensurability in terms of $r$, i.e., if there is no natural number $r$ satisfying
(2), the motion is not periodic. The important case or condition for which $\mathrm{T}=2 \pi / \omega_{\mathrm{D} 1}$ is that $\omega_{\mathrm{D} 1}$ and $\omega_{\mathrm{D} 2}$ are integer multiples of each other or that $\mathrm{r}=\mathrm{q}$ and $\mathrm{p}=1$.

The equation of motion of the cantilever can be reduced $^{5}$ to m modes and two external drives as

$$
\begin{aligned}
& \frac{k_{(m)}}{\omega_{(m)}^{2}} \ddot{z}_{(m)}(t)+\frac{k_{(m)}}{Q_{(m)} \omega_{(m)}} \dot{z}_{(m)}(t)+k_{(m)} z_{(m)} \\
& \quad=F_{0 D 1} \sin \left(\omega_{D 1} t\right)+F_{0 D 2} \sin \left(\omega_{D 2} t\right)+F_{t s},
\end{aligned}
$$

where $\mathrm{k}_{(\mathrm{m})}, \mathrm{Q}_{(\mathrm{m})}$, and $\omega_{(\mathrm{m})}$ are the spring constant, quality factor, and natural frequency of the $\mathrm{m}$ eigenmode. $\mathrm{F}_{0 \mathrm{D} 1}$ $\approx \mathrm{F}_{0(1)}$ and $\mathrm{F}_{0 \mathrm{D} 2} \approx \mathrm{F}_{0(2)}$ are the magnitudes of the two external drives acting at or near $\omega_{(1)}$ and $\omega_{(2)}$, respectively. $F_{t s}$ is the unrestricted tip-sample force and $\mathrm{z}_{(\mathrm{m})}$ is the modal tip position $^{38}$ that can be expanded in terms of modal higher harmonics $^{39}$

$$
z_{(m)} \approx z_{(m) 0}+\sum_{n>0}^{N} A_{(m) n} \sin \left(n \omega_{F} t+\phi_{(m) n}\right) .
$$

Conservative interactions associated with each mode can be described in terms of the modal Virial $\mathrm{V}_{(\mathrm{m})} 28,37$

$$
\begin{aligned}
V_{(m)} & =\frac{1}{T} \int_{t=t^{\prime}}^{t=t^{\prime}+T} F_{t s} z_{(m)} d t \\
& =-\frac{1}{2} F_{0(m)} A_{(m) n} \cos \phi_{(m) n}+H D\left(V_{(m)}\right) .
\end{aligned}
$$

The last term on the right is the harmonic distortion HD in Virial for mode $\mathrm{m}$, i.e., all frequencies except for $\mathrm{n}$, and the term before it coincides with the Virial related to the frequency of interest $\mathrm{n}$. It is important to note that the availability of a fundamental frequency $\omega_{\mathrm{F}}$ or periodicity, as expressed by the relationship in (2) and the expressions in (1) and (4), is necessary in order to invoke orthogonality in the integral of the product $\mathrm{F}_{\mathrm{ts}} \mathrm{Z}_{(\mathrm{m})}$ in (5). Then, periodicity ${ }^{40}$ leads to the important simplification

$$
V_{(m) n} \approx V_{n}=-\frac{1}{2} F_{0 n} A_{n} \cos \phi_{n} .
$$

The above expression is particularly relevant in bimodal AFM for the two monitored frequencies $\omega_{\mathrm{D} 1}=\mathrm{p} \omega_{\mathrm{F}}$ and $\omega_{\mathrm{D} 2}=\mathrm{q} \omega_{\mathrm{F}}$, i.e., harmonics $\mathrm{n}=\mathrm{p}(\mathrm{m}=1)$ and $\mathrm{q}(\mathrm{m}=2)$. Similarly, for the energy transfer $\mathrm{E}_{\mathrm{T}(\mathrm{m})}$ of mode $\mathrm{m}$ (Ref. 37)

$$
\begin{aligned}
E_{T(m)}= & \pi n F_{0(m)} A_{(m) n} \sin \phi_{(m) n}+\pi \frac{k_{(m)}}{Q_{(m)}} \frac{\omega_{S H}}{\omega_{(m)}} n^{2} A_{(m) n}^{2} \\
& +H D\left(E_{T(m)}\right),
\end{aligned}
$$

where, as before, HD stands for harmonic distortion but this time related to energy transfer. Again, provided the signal is periodic as described by (1), (2) and (4), (7) reduces to

$$
E_{T(m) n} \approx E_{T n}=\pi n \frac{k_{(m)} A_{0 n} A_{n}}{Q_{(m)}}\left[\sin \phi_{n}-\frac{A_{n}}{A_{0 n}} \frac{n \omega_{S H}}{\omega_{(m)}}\right],
$$


where we again focus on $\omega_{\mathrm{D} 1}=\mathrm{p} \omega_{\mathrm{F}}$ and $\omega_{\mathrm{D} 2}=\mathrm{q} \omega_{\mathrm{F}}$, i.e., $\mathrm{n}=\mathrm{p}$ and $\mathrm{q}$ as before. The combination of (6) and (8) leads to the fundamental expression of phase shift $\phi_{\mathrm{n}}{ }^{28}$

$$
\phi_{n} \approx \tan ^{-1}\left[-\frac{\frac{E_{T n} Q_{(m)}}{2 \pi n}+\frac{1}{2} k_{(m)} A_{n}^{2}}{V_{n} Q_{(m)}}\right] .
$$

Finally, the energy irreversibly lost to the tip-sample interaction $E_{\text {dis }}$ is the contribution of energy transfer from all modes, ${ }^{7,37}$ i.e., in bimodal, the first two modes, as

$$
E_{d i s}=E_{T(1)}+E_{T(2)} .
$$

Next, the above theory is submitted to the test of numerical integration of (3). For the tip-sample forces, the first expression relates to the long range forces and $F_{t s}$ is defined by the Hamaker constant $\mathrm{H}^{26}$ the effective tip radius $\mathrm{R}$, and the tip sample distance d (Ref. 20)

$$
F_{t s}(d)=-\frac{R H}{6 d^{2}} \quad \mathrm{a}_{0}<\mathrm{d},
$$

where $\mathrm{a}_{0}(\approx 0.165 \mathrm{~nm})$ is an intermolecular distance. In the short range $\mathrm{d} \leq \mathrm{a}_{0}, \mathrm{~F}_{\mathrm{ts}}$ is modelled with ${ }^{20,26}$

$$
F_{t s}(d)=-\frac{R H}{6 a_{0}^{2}}+\frac{4}{3} E^{*} \sqrt{R} \delta^{3 / 2} \quad \mathrm{a}_{0} \geq \mathrm{d},
$$

where $\mathrm{d}$ and the tip-sample deformation $\delta$ are related by $\delta=\mathrm{a}_{0}-\mathrm{d}$. $\mathrm{E}^{*}(\approx 1 \mathrm{GPa})$ is the effective Young modulus in the contact. Numerical results are presented in Fig. 2. The frequencies (horizontal axis) have been normalized with $\mathrm{f}_{\mathrm{D} 1}$. The generic parameters are: $A_{0 p}=20 \mathrm{~nm}$ (free amplitude mode 1), $\mathrm{A}_{0 \mathrm{q}}=1 \mathrm{~nm}$ (free amplitude mode 2), $\mathrm{A}_{\mathrm{p}}=10 \mathrm{~nm}$ (set-point), $\mathrm{k}_{(1)}=2 \mathrm{~N} / \mathrm{m}, \mathrm{k}_{(2)}=80 \mathrm{~N} / \mathrm{m}, \mathrm{Q}_{(1)}=100, \mathrm{Q}_{(2)}=600, \mathrm{H}=2.1$ $\times 10^{-20} \mathrm{~J}$, and $\mathrm{R}=5 \mathrm{~nm}$. These conditions led to the average force remaining positive throughout in the simulations, i.e., repulsive regime. ${ }^{26}$ Only two conditions are presented in Fig. 2 for clarity and simplicity. For the first one, $r \neq q$ and $p \neq 1$ (Figs. 2(a) and 2(b)). This is the case for which the drive frequencies are not multiples of each other and a fundamental frequency lower than $\omega_{\mathrm{D} 1}$ is present. In particular, here $\mathrm{q}=31$, $\mathrm{p}=5$, and $\mathrm{r}=31 / 5$ implying that the fundamental frequency $\omega_{\mathrm{F}}$ is 5 times lower than the first drive $\omega_{\mathrm{D} 1}$ and that the $\mathrm{T}$ is 5 times longer than the period of the first drive. The presence of harmonics multiple of $\omega_{\mathrm{F}}=\omega_{\mathrm{D} 1} / 5$ is observed in both Fig. 2(a) and the zoom in Fig. 2(b). The second case shown in the figure (Figs. 2(c) and 3(d)) exemplifies the consequences of driving with a second frequency multiple of the first drive, i.e., $\omega_{\mathrm{D} 2}=\mathrm{q} \omega_{\mathrm{D} 1}$; here, $\omega_{\mathrm{D} 2}=6 \omega_{\mathrm{D} 1}$. In this case, all higher harmonics are exact multiples of $\omega_{\mathrm{D} 1}$ and the period is $\mathrm{T}=2 \pi /$ $\omega_{\mathrm{D} 1}$ in agreement with the theory above. We note that driving with frequencies that are exact multiples of each other does not require that cantilevers are harmonic but simply depends on the choice of drives. From (2), it is required that $\mathrm{p}=1$ or $\omega_{\mathrm{F}}=\omega_{\mathrm{D} 1}$ as we verified in all cases in our simulations (not all data shown). This means that irrespective of the characteristics of the cantilever, the period $\mathrm{T}$ can be controlled by the choice of drive frequencies alone as also verified experimentally (see below). In terms of $\mathrm{T}$ in Fig. 2 , when $\mathrm{r}=6.2, \mathrm{~T} \approx 71 \mu \mathrm{s}$ while for $\mathrm{r}=6, \mathrm{~T} \approx 14 \mu \mathrm{s}$. The effects of the two different scenarios illustrated in Fig. 2 in terms of the fundamental expressions in bimodal AFM (5)-(10) are provided quantitatively in Table I. It is particularly interesting to note that both the Virial (6) and the energy transfer (8) expression for the second mode, i.e., $\mathrm{n}=\mathrm{q}$, change in sign depending on $\mathrm{r}$.

We further verified in simulations that incommensurability, i.e., no natural number $r$ satisfying (2), led to many peaks at even smaller frequencies than those in Figs. 2(a) and 2(b). In general, as r, p, and q increase, $\omega_{\mathrm{F}}$ decreases and $\mathrm{T}$ increases as predicted by (2). ${ }^{41}$

Next, experimental waveforms (Fig. 3) of the free and engaged cantilever and the corresponding Fast Fourier Transforms (FFTs) (Fig. 4) were acquired in order to experimentally validate the theoretical predictions. An Asylum Research (Cypher) AFM was used in the experiments. A cantilever with $\mathrm{k}_{(1)} \approx 1.7 \mathrm{~N} / \mathrm{m}$ and $\mathrm{f}_{(1)} \approx 73 \mathrm{kHz}$ was employed (OLYMPUS AC240TS) and the parameters were $\mathrm{A}_{0(1)}$ $\approx 56 \mathrm{~nm}_{\text {s }} \mathrm{A}_{0(2)} \approx 1 \mathrm{~nm}$, and set point $\mathrm{A}_{(1)} \equiv \mathrm{A}_{\mathrm{sp}} \approx 0.6 \mathrm{~A}_{0(1)}$. Three relationships were selected for $\mathrm{f}_{\mathrm{D} 1}$ and $\mathrm{f}_{\mathrm{D} 2}$ as parameterized by $r=f_{D 2} / f_{D 1}$ as in (2): $r=5.9, r=6$, and $r=6.2$ in Fig. 3. The cantilever was anharmonic with $f_{(1)}=72.715 \mathrm{kHz}$ and $\mathrm{f}_{(2)}=434.814 \mathrm{kHz} ; \mathrm{f}_{(2)} / \mathrm{f}_{(1)}=5.98$.
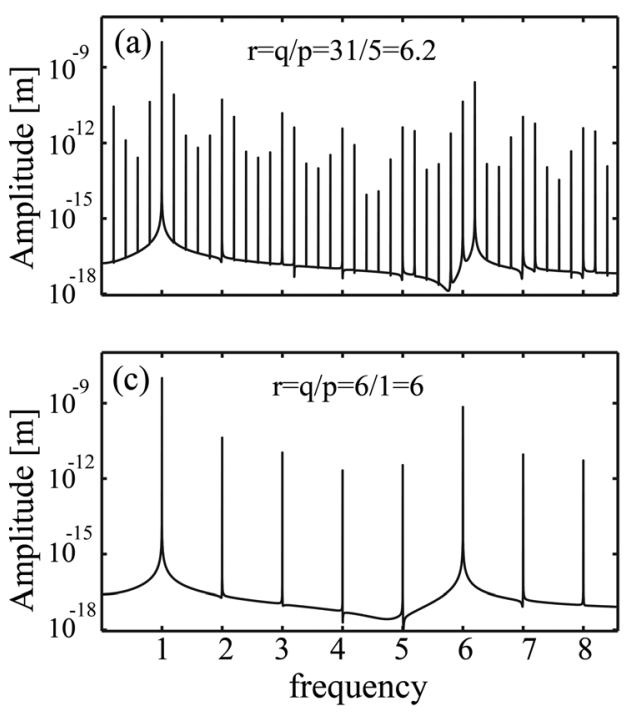
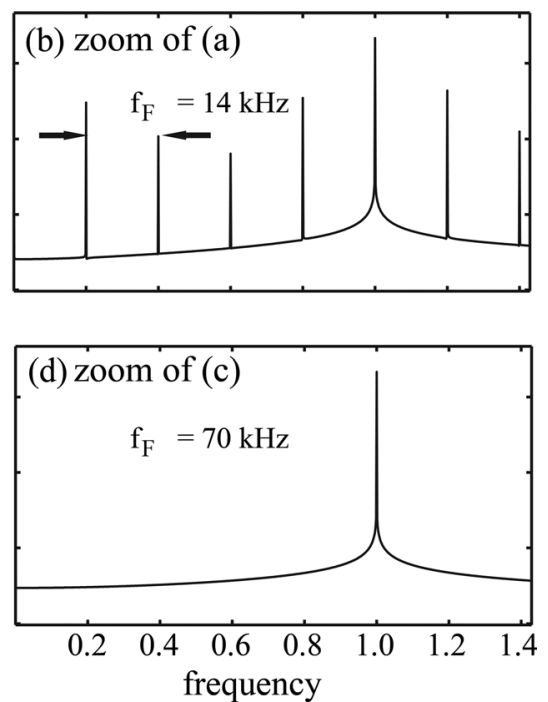

FIG. 2. Examples of the frequency spectrum (amplitude/frequency) for an interacting tip depending on the relationship between drive frequencies as parameterized by $r=q / p$. The panels on the right correspond to zooms of the panels on the left and the horizontal axis has been normalized by the first drive frequency $f_{D 1}$. 


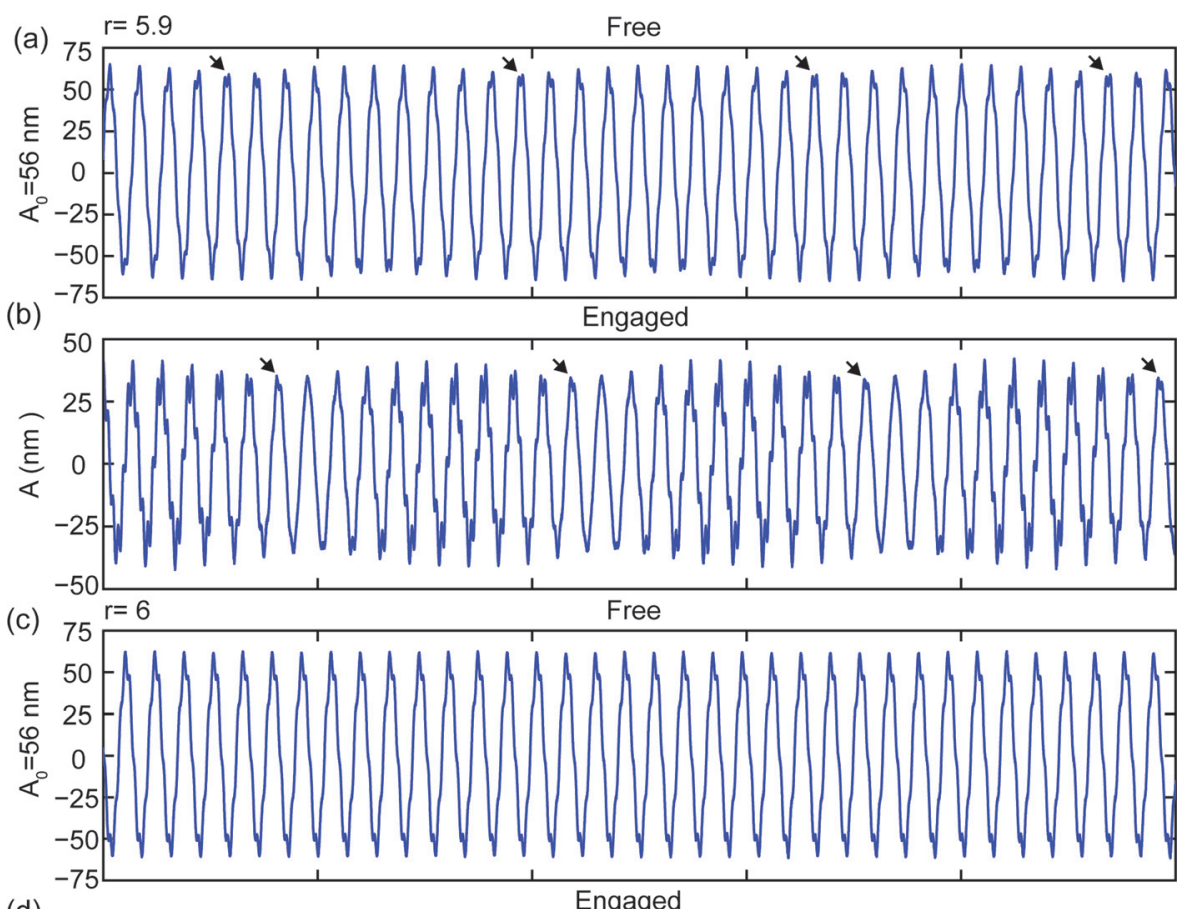

(d)
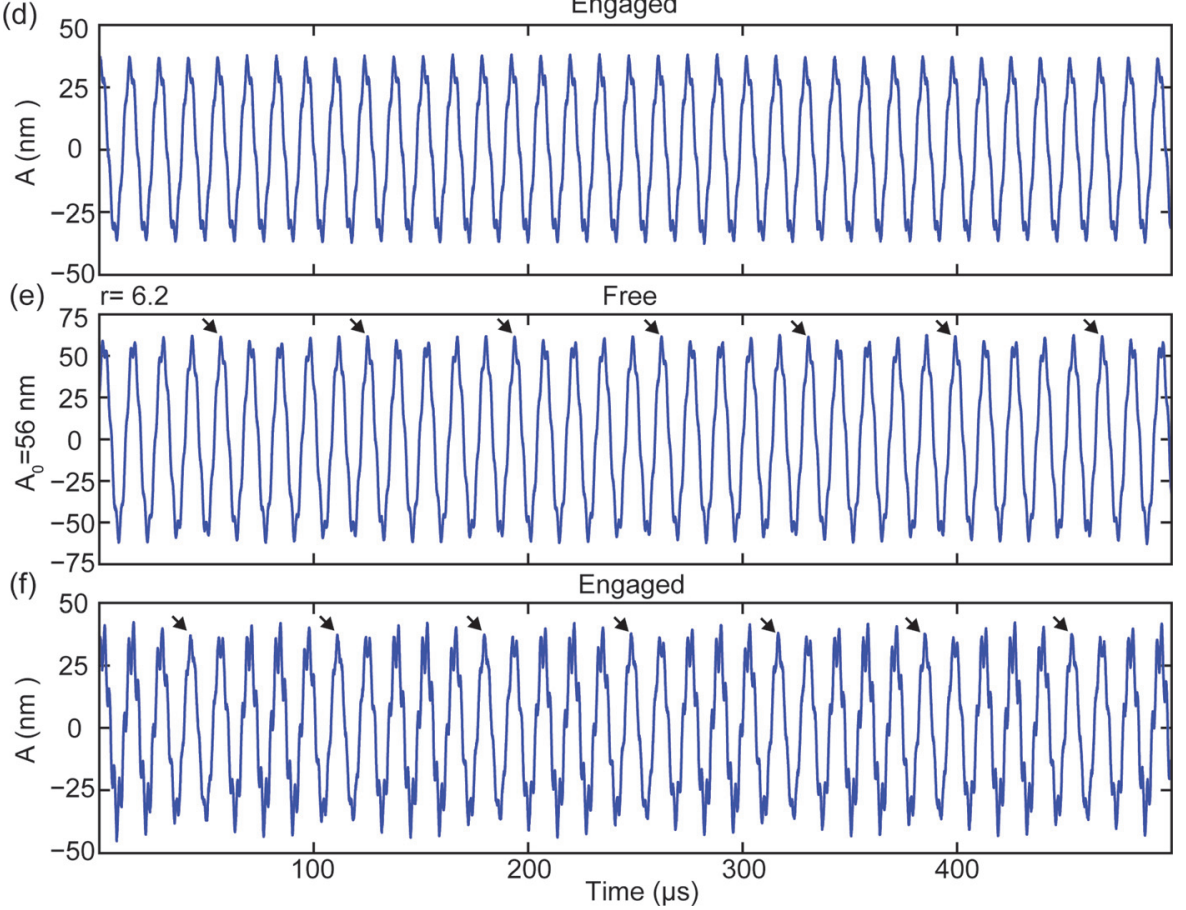

FIG 3. Experimental waveforms obtained on a mica sample for a free and engaged cantilever for (a) and (b) $\mathrm{r}=5.9$, (c) and (d) $\mathrm{r}=6$, and (e) and (f) $r=6.2$. Arrows indicate period.
For $r=5.9$, the waveforms (time domain) of both the free and engaged cantilever display a pattern that repeated every 10 periods of the first drive (Figs. 3(a) and 3(b)) as indicated with arrows. For $r=6$ (Figs. 3(c) and 3(d)), the waveforms of the free and engaged cantilever displayed a pattern that repeated every period of the first drive. Finally, for $r=6.2$ (Figs. 3(e) and 3(f)), the waveforms displayed a pattern that repeated every five periods of the first drive. Note that when $r=5.9$ and $=6.2$, one can distinguish a wave packet appearing in the waveforms for both the free and engaged cantilever.

TABLE I. Numerical values of some of the fundamental expressions in bimodal AFM as discussed in this work and obtained in the simulations from Fig. 2. The number of the expressions employed is specified and the numerical values given as obtained when acquiring an FFT in the simulations. The last column corresponds to the value of phase shift for $\mathrm{n}=\mathrm{q}$ (monitored phase shift near the second mode) resulting from the FFT in simulations (Sim).

\begin{tabular}{|c|c|c|c|c|c|c|c|c|c|}
\hline & $\begin{array}{l}\text { Equation (5) } \\
\mathrm{V}_{(1)}(\mathrm{eV})\end{array}$ & $\begin{array}{l}\text { Equation (5) } \\
\mathrm{V}_{(2)}(\mathrm{eV})\end{array}$ & $\begin{array}{c}\text { Equation (7) } \\
\mathrm{E}_{\mathrm{T}(1)}(\mathrm{eV})\end{array}$ & $\begin{array}{l}\text { Equation (7) } \\
\mathrm{E}_{\mathrm{T}(2)}(\mathrm{eV})\end{array}$ & $\begin{array}{l}\text { Equation (10) } \\
\mathrm{E}_{\mathrm{dis}}(\mathrm{eV})\end{array}$ & $\begin{array}{c}\text { Equation (6) } \\
\mathrm{V}_{\mathrm{q}}(\mathrm{eV})\end{array}$ & $\begin{array}{c}\text { Equation (8) } \\
\mathrm{E}_{\mathrm{Tq}}(\mathrm{eV})\end{array}$ & $\begin{array}{l}\text { Equation (9) } \\
\Phi_{q}(\operatorname{deg})\end{array}$ & $\begin{array}{c}(\operatorname{Sim}) \Phi_{\mathrm{q}} \\
\quad(\mathrm{deg})\end{array}$ \\
\hline$r=31 / 5$ & -10.83 & -0.05 & 0.23 & -0.23 & 0.00 & -0.10 & 0.03 & 14.80 & 14.81 \\
\hline$r=6 / 1$ & -9.53 & -0.31 & 11.29 & -11.29 & 0.00 & 0.32 & -7.61 & 171.32 & 171.32 \\
\hline
\end{tabular}



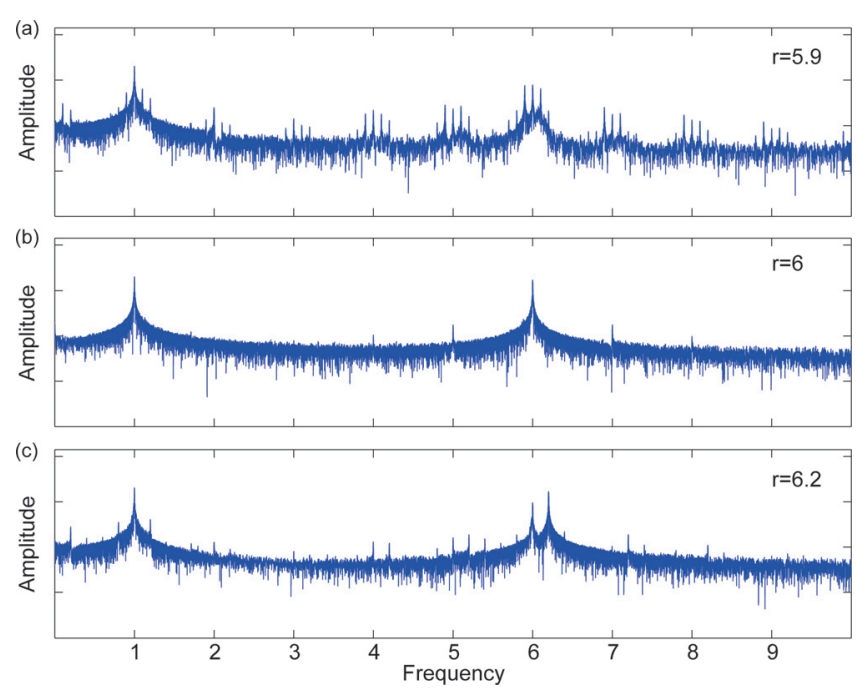

FIG. 4. Experimental FFTs obtained for an engaged cantilever for (a) $r=5.9$, (b) $r=6$, and (c) $r=6.2$. The data correspond to the waveforms in Fig. 3 for the engaged cantilever.

The FFTs (frequency domain) for the data in Fig. 3 are shown in Fig. 4. In the case of $r=5.9$, the fundamental frequency is $f_{F}=7.3 \mathrm{kHz}=0.1 f_{D 1}$. When $r=6$, the fundamental frequency is $f_{S H}=73 \mathrm{kHz}=f_{D 1}$. When $r=6.2$, the fundamental frequency is $\mathrm{f}_{\mathrm{SH}}=14.6 \mathrm{kHz}=0.2 \mathrm{f}_{\mathrm{D} 1}$. Harmonics appear as multiples of $f_{F}$ in all counts in Fig. 4 as predicted. At this point, we would like to comment on the fact that the period of the signal, or otherwise the fundamental frequency $\mathrm{f}_{\mathrm{F}}$, remained unchanged when the cantilever interacted with the surface. That is, the arbitrary choice or selection of frequencies by the user for the free cantilever giving place to the ratio $\mathrm{r}$ being rational, or otherwise the presence of a fundamental frequency $f_{F}$, remained unaltered when the cantilever interacted in the results presented here. In this sense, while it could be argued that it is expected that the fundamental period or frequency of a linear system, i.e., the unperturbed or free cantilever, can be selected arbitrarily with an appropriate choice of external drives, ${ }^{36}$ it has also been shown in the literature that the fundamental period can change when the cantilever interacts with the nonlinear tip-surface forces even when employing a single drive. ${ }^{42,43}$ Thus, we believe that it is remarkable that in the more complex set-up of bimodal AFM, and more specifically for the cases explored in this work, the fundamental frequency selected by the user remained unaltered throughout. The periodicity of the system during tip-sample interaction and the presence of multiple accessible frequencies might further offer potential for multi-parametric AFM. ${ }^{8,40,44}$

\section{CONCLUSIONS}

In summary, the existence of a fundamental period of oscillation or repeat in bimodal AFM depends on the relationship between external drive frequencies and can be tuned by the user. Periodicity also simplifies the theory of bimodal AFM and leads to simple analytical expressions. The possibility to induce periodic motion and derive simple analytical expressions should further assist in developing quantitative forms of bimodal AFM that rely on steady-state oscillation, periodicity, and/or conservation principles.

${ }^{1}$ G. Binnig, C. F. Quate, and C. Gerber, Phys. Rev. Lett. 56, 930 (1986).

${ }^{2}$ T. R. Albrecht, P. Grutter, D. Horne, and D. Rugar, J. Appl. Phys. 69, 668 (1991).

${ }^{3}$ Y. Martin, C. C. Williams, and H. K. Wickramasinghe, J. Appl. Phys. 61, 4723 (1987).

${ }^{4}$ Q. Zhong, D. Inniss, K. Kjoller, and V. B. Elings, Surface Science 290, L688 (1993).

${ }^{5}$ T. Rodriguez and R. Garcia, Appl. Phys. Lett. 84, 449 (2004).

${ }^{6}$ Y. Maeda, T. Matsumoto, and T. Kawai, Appl. Surf. Sci. 140, 400 (1999).

${ }^{7}$ S. An, S. D. Solares, S. Santos, and D. Ebeling, Nanotechnology 25, 475701 (2014).

${ }^{8}$ D. Forchheimer, R. Forchheimer, and D. B. Haviland, Nat. Commun. 6, 6270 (2015).

${ }^{9}$ O. Sahin, C. Quate, O. Solgaard, and F. J. Giessibl, Higher-Harmonic Force Detection in Dynamic Force Microscopy, Springer Handbook of Nanotechnology (Springer-Verlag, Berlin-Heidelberg, 2007).

${ }^{10}$ M. Stark, R. W. Stark, W. M. Heckl, and R. Guckenberger, Proc. Natl. Acad. Sci. U. S. A. 99, 8473 (2002).

${ }^{11}$ S. Basak and A. Raman, Appl. Phys. Lett. 91, 064107 (2007).

${ }^{12}$ T. R. Rodríguez and R. García, Appl. Phys. Lett. 80, 1646 (2002).

${ }^{13}$ D. Martinez-Martin, E. T. Herruzo, C. Dietz, J. Gomez-Herrero, and R. Garcia, Phys. Rev. Lett. 106, 198101 (2011).

${ }^{14}$ D. Kiracofe, A. Raman, and D. Yablon, Beilstein J. Nanotechnol. 4, 385 (2013).

${ }^{15}$ S. D. Solares and G. Chawla, J. Appl. Phys. 108, 054901 (2010).

${ }^{16}$ S. Kawai, T. Glatzel, S. Koch, B. Such, A. Baratoff, and E. Meyer, Phys. Rev. Lett. 103, 220801 (2009).

${ }^{17}$ R. Garcia and R. Proksch, Eur. Polym. J. 49, 1897 (2013).

${ }^{18}$ E. T. Herruzo, A. P. Perrino, and R. Garcia, Nat. Commun. 5, 3126 (2014).

${ }^{19}$ R. Proksch, Appl. Phys. Lett. 89, 113121 (2006).

${ }^{20}$ A. S. Paulo and R. Garcia, Phys. Rev. B 64, 193411 (2001).

${ }^{21}$ R. Stark, Appl. Phys. Lett. 94, 063109 (2009).

${ }^{22}$ I. Chakraborty and D. G. Yablon, Nanotechnology 24, 475706 (2013).

${ }^{23}$ S. Santos, V. Barcons, J. Font, and A. Verdaguer, Beilstein J. Nanotechnol. 5, 268 (2014).

${ }^{24}$ H. V. Guzman and R. Garcia, Beilstein J. Nanotechnol. 4, 852 (2013).

${ }^{25}$ R. W. Stark, G. Schitter, and A. Stemmer, Phys. Rev. B 68, 085401 (2003).

${ }^{26}$ R. Garcia and A. San Paulo, Phys. Rev. B 60, 4961 (1999).

${ }^{27}$ J. P. Cleveland, B. Anczykowski, A. E. Schmid, and V. B. Elings, Appl. Phys. Lett. 72, 2613 (1998).

${ }^{28}$ J. R. Lozano and R. Garcia, Phys. Rev. Lett. 100, 076102 (2008).

${ }^{29}$ O. Sahin, C. Quate, O. Solgaard, and A. Atalar, Phys. Rev. B 69, 165416 (2004).

${ }^{30}$ D. Kiracofe and A. Raman, J. Appl. Phys. 107, 033506 (2010).

${ }^{31}$ R. Steidel, An Introduction to Mechanical Vibrations, 3rd ed. (John Wiley and Sons, 1989).

${ }^{32}$ H.-J. Butt and M. Jaschke, Nanotechnology 6, 1 (1995).

${ }^{33}$ R. Garcia and E. T. Herruzo, Nat. Nanotechnol. 7, 217 (2012).

${ }^{34}$ M. Loganathan and D. A. Bristow, Rev. Sci. Instrum. 85, 043703 (2014).

${ }^{35}$ G. Saraswat and M. V. Salapaka, Appl. Phys. Lett. 102, 173108 (2013).

${ }^{36}$ G. P. Tolstov and R. A. Silverman, Fourier Series (Dover Publications, Inc., New York, 1976).

${ }^{37}$ S. Santos, Appl. Phys. Lett. 104, 143109 (2014).

${ }^{38}$ J. Melcher, C. Carrasco, X. Xu, J. L. Carrascosa, J. Gomez-Herrero, P. J. de Pablo, and A. Raman, Proc. Natl. Acad. Sci. U. S. A. 106, 13655 (2009).

${ }^{39}$ A. F. Payam, J. R. Ramos, and R. Garcia, ACS Nano 6, 4663 (2012).

${ }^{40}$ S. Santos and V. Barcons, e-print arXiv.org/abs/1407.3165.

${ }^{41}$ See supplementary material at http://dx.doi.org/10.1063/1.4927733 for $\mathrm{p}=100$.

${ }^{42}$ D. Kiracofe and A. Raman, J. Appl. Phys. 108, 034320 (2010).

${ }^{43}$ M. Chiesa, K. R. Gadelrab, A. Verdaguer, J. Segura, V. Barcons, N. H. Thomson, M. A. Phillips, M. Stefancich, and S. Santos, EPL 99, 56002 (2012).

${ }^{44}$ M. Chiesa et al., J. Phys. Chem. Lett. 3, 2125 (2012). 